\title{
Associations between the erythropoiesis- stimulating agent resistance index and the geriatric nutritional risk index of maintenance hemodialysis patients and increased mortality
}

\author{
Masayuki Okazaki ${ }^{1}$, Mizuki Komatsu', Shunji Shiohira², Hiroshi Kataoka², Ken Tsuchiya ${ }^{2}$, Hiroshi Kawaguchi ${ }^{1}$ and \\ Kosaku Nitta ${ }^{2^{*}}$
}

\begin{abstract}
Background: There may be an association between the responsiveness to erythropoiesis-stimulating agent (ESA) and the nutritional status on mortality of maintenance hemodialysis (MHD) patients. Therefore, we assessed the associations of geriatric nutritional risk index (GNRI) and ESA resistance index (ERI) in mortality in MHD patients.

Methods: A total of 298 MHD patients were enrolled. Unadjusted and adjusted Cox regression analyses for all-cause mortality were performed. Odds ratios were estimated using a logistic regression model for the association between the GNRI and ERI and mortality, adjusting for potential confounders.

Results: During the follow-up period of $34.6 \pm 6.1$ months, 51 died with 31 cardiovascular deaths. Receiver operating curve analyses indicated the cutoff values of GNRI and ERI for mortality were 94.9 and 13.5, respectively. When subjects were stratified by ERI and GNRI values into four groups, those who had low GNRI and high ERI were associated with the highest risk of mortality among the four groups (log-rank $p<0.001$ ).
\end{abstract}

Conclusions: High ERI and low GNRI were associated with an increased risk of all-cause mortality.

Keywords: Geriatric nutritional risk index, Erythropoiesis-stimulating agent, Hyporesponsiveness, Hemodialysis, Mortality

\section{Background}

Protein-energy wasting (PEW) is a major problem in endstage renal disease (ESRD) patients, and its reported prevalence has ranged from about 20 to $78 \%$ [1]. Since nutritional risk is one of the strongest predictors of increased mortality in maintenance hemodialysis (MHD) patients, and evaluation of nutritional status and nutritional management are essential clinical procedures for managing MHD patients $[2,3]$, it is necessary to regularly screen patients for PEW by making a variety of measurement with a variety of measures [4]. The geriatric nutritional risk index (GNRI) is

\footnotetext{
* Correspondence: knitta@kc.twmu.ac.jp

2Department of Medicine, Kidney Center, Tokyo Women's Medical University, Shinjuku-ku, Tokyo, Japan

Full list of author information is available at the end of the article
}

a very simple and objective method of assessing nutritional status in a number of pathological conditions that is based on body weight, height, and serum albumin levels. Some studies have investigated the reliability of the GNRI as a tool for assessing malnutrition in HD patients [5] and predicting their overall mortality $[6,7]$.

The development of erythropoiesis-stimulating agents (ESAs) has resulted in substantial health benefits for ESRD patients, including improved quality of life, reduced need for blood transfusion, and increased exercise capacity. Unfortunately, however, a considerable proportion of ESRD patients exhibit a suboptimal hematologic response to ESAs as evidenced by the persistence of anemia despite adequate dosing or by the need for high ESA doses to achieve the recommended hemoglobin 
( $\mathrm{Hb})$ target [8]. ESA doses and the Hb levels achieved in HD patients have been found to be associated with mortality [9], and hyporesponsiveness to ESA therapy is known to predict a poorer outcome [10]. Both high-dose ESA therapy and low $\mathrm{Hb}$ levels may contribute to poorer outcomes of HD patients who are hyporesponse to ESAs $[11,12]$.

The aim of the present study was to clarify the relationship between both the GNRI and responsiveness to ESA of MHD patients and their long-term mortality.

\section{Methods}

\section{Study design}

This was a prospective, observational cohort study conducted at a single center in Japan. The subjects were recruited from among patients who had been routinely dialyzed via an arteriovenous fistula in the dialysis unit of the Jyoban Hospital for at least 6 months. The Institutional Review Board of the Jyoban Hospital approved all study protocols (no. 11), and the protocols were carried out in accordance with the Declaration of Helsinki guidelines regarding ethical principles for medical research involving human subjects. Written informed consent was obtained from every subject.

HD patients with a malignancy, active inflammation, liver cirrhosis, gastrointestinal bleeding, cardiac valvular disease, or severe illness were excluded from participation and were transferred to another dialysis unit for intensive care. The patients who were enrolled as subjects $(n=298)$ underwent stable regular HD with a bicarbonate dialysate. Their underlying diseases of ESRD were as follows: diabetic nephropathy $(n=144)$, chronic glomerulonephritis $(n=76)$, hypertensive nephrosclerosis $(n=70)$, chronic pyelonephritis $(n=3)$, and unknown $(n=5)$. Autosomal-dominant polycystic kidney disease patients often have higher $\mathrm{Hb}$ levels and lower ESA requirements than ESRD patients with other underlying diseases, and they were excluded.

All patients were on thrice-weekly HD sessions. Blood pressure (BP) was measured with a mercury sphygmomanometer with the patient in the supine position after resting for 10 to $15 \mathrm{~min}$, and mean values for the 1month period preceding enrollment were used in the

Table 1 Baseline characteristics of the study population

\begin{tabular}{|c|c|c|c|c|c|c|}
\hline & \multirow[t]{2}{*}{ All patients $(n=298)$} & \multicolumn{4}{|l|}{ ERI quartiles } & \multirow[t]{2}{*}{$P$ value } \\
\hline & & $\mathrm{Q} 1(n=74)$ & $\mathrm{Q} 2(n=75)$ & Q3 $(n=75)$ & Q4 $(n=74)$ & \\
\hline Age, years & $66.0 \pm 13.1$ & $61.7 \pm 13.0$ & $65.3 \pm 11.8$ & $66.8 \pm 14.1$ & $70.2 \pm 12.2$ & 0.0010 \\
\hline Male, \% & $187(62.8 \%)$ & $54(73.0 \%)$ & 49 (65.3\%) & 47 (62.7 \%) & 37 (50.0 \%) & 0.0340 \\
\hline Diabetes, \% & $144(48.3 \%)$ & 39 (52.7\%) & 38 (50.7 \%) & 34 (45.3\%) & $33(44.6 \%)$ & 0.7023 \\
\hline Cardiovascular disease, \% & 78 (26.2 \%) & 22 (29.7\%) & $21(28.0 \%)$ & 18 (24.0\%) & 17 (23.0 \%) & 0.7555 \\
\hline Dialysis vintage, years & $3.3(1.2-6.7)$ & $2.8(1.1-5.6)$ & $3.5(1.1-8.1)$ & $3.6(1.1-7.6)$ & $3.5(1.3-6.8)$ & 0.6318 \\
\hline $\mathrm{Kt} / \mathrm{N}$ & $1.4 \pm 0.2$ & $1.4 \pm 0.2$ & $1.4 \pm 0.3$ & $1.4 \pm 0.2$ & $1.4 \pm 0.3$ & 0.5231 \\
\hline Systolic BP, mmHg & $153.1 \pm 17.2$ & $154.6 \pm 17.6$ & $149.4 \pm 17.6$ & $155.5 \pm 17.2$ & $153.0 \pm 16.2$ & 0.1354 \\
\hline Diastolic BP, mmHg & $79.8 \pm 11.5$ & $82.6 \pm 11.8$ & $77.9 \pm 11.1$ & $80.6 \pm 12.2$ & $78.0 \pm 10.4$ & 0.0357 \\
\hline Pulse pleasure, $\mathrm{mmHg}$ & $73.3 \pm 13.1$ & $72.0 \pm 13.1$ & $71.5 \pm 13.2$ & $74.9 \pm 12.4$ & $75.0 \pm 13.5$ & 0.2061 \\
\hline Body mass index, $\mathrm{kg} / \mathrm{m}^{2}$ & $22.0 \pm 3.5$ & $23.0 \pm 3.2$ & $22.5 \pm 3.7$ & $21.8 \pm 3.6$ & $20.6 \pm 2.9$ & 0.0002 \\
\hline Albumin, g/dl & $3.7 \pm 0.3$ & $3.7 \pm 0.3$ & $3.7 \pm 0.3$ & $3.6 \pm 0.3$ & $3.6 \pm 0.4$ & 0.2525 \\
\hline $\mathrm{nPCR}, \mathrm{g} / \mathrm{kg} / \mathrm{dl}$ & $0.8 \pm 0.2$ & $0.8 \pm 0.2$ & $0.9 \pm 0.2$ & $0.8 \pm 0.2$ & $0.8 \pm 0.2$ & 0.7969 \\
\hline $\mathrm{CRP}, \mathrm{mg} / \mathrm{dl}$ & $0.8 \pm 0.0$ & $0.3 \pm 0.7$ & $0.3 \pm 0.6$ & $0.4 \pm 0.8$ & $0.5 \pm 0.9$ & 0.4315 \\
\hline Hemoglobin, g/dl & $10.9 \pm 1.0$ & $11.1 \pm 0.8$ & $11.2 \pm 0.8$ & $10.7 \pm 1.0$ & $10.4 \pm 1.1$ & $<0.0001$ \\
\hline Total cholesterol, mg/dl & $150.6 \pm 30.4$ & $150.9 \pm 29.7$ & $151.7 \pm 32.9$ & $152.2 \pm 32.9$ & $147.6 \pm 25.9$ & 0.7929 \\
\hline Calcium (corrected), mg/dl & $9.1 \pm 0.6$ & $9.1 \pm 0.6$ & $9.1 \pm 0.6$ & $9.2 \pm 0.6$ & $9.1 \pm 0.5$ & 0.2019 \\
\hline Phosphorus, mg/dl & $4.9 \pm 1.6$ & $5.0 \pm 1.4$ & $5.4 \pm 1.7$ & $4.4 \pm 1.6$ & $4.7 \pm 1.6$ & 0.0014 \\
\hline Intact-PTH, pg/ml & $94(54-168)$ & 109 (67-179) & $102(47-156)$ & $75(49-151)$ & $110(46-183)$ & 0.2870 \\
\hline GNRI & $96.3 \pm 8.7$ & $99.0 \pm 8.1$ & $97.6 \pm 9.2$ & $95.4 \pm 8.6$ & $93.3 \pm 8.0$ & 0.0003 \\
\hline Ferritin, ng/ml & $63(24-125)$ & $56(25-133)$ & $65(21-125)$ & $44(21-108)$ & $72(41-144)$ & 0.0893 \\
\hline TSAT, $\%$ & $21.6 \pm 10.3$ & $24.7 \pm 9.5$ & $22.5 \pm 10.7$ & $21.0 \pm 10.2$ & $18.3 \pm 10.0$ & 0.0016 \\
\hline ERI, IU/kg/week/Hb & $8.2(5.0-13.1)$ & $3.6(2.7-4.1)$ & $6.2(5.4-7.0)$ & $10.7(9.3-11.7)$ & $17.5(14.8-20.7)$ & $<0.0001$ \\
\hline
\end{tabular}

Continuous values are expressed as means \pm SD. Count data are expressed as percentages

Abbreviations: $B P$ blood pressure, $n P C R$ normalized protein catabolic rate, $C R P$ C-reactive protein, $P T H$ parathyroid hormone, TSAT transferrin saturation, ERI erythropoiesis resistance index 
statistical analysis. Dry weight was targeted to achieve a normotensive edema-free state. History of previous cardiovascular disease was collected from the medical records. Diabetes was recorded as a history or presence of diabetes and/or a fasting plasma glucose concentration $>126 \mathrm{mg} / \mathrm{dl}$ or HbA1c concentration $>6.5 \%$ or prescription of glucose-lowering agents.

The study period was divided into two phases to evaluate the time-dependent risk of mortality based on subjects' longitudinal dialysis status. The first phase (12 months) was used to collect laboratory data to evaluate longitudinal dialysis status. The second phase (the subsequent 2 years) was used as a means of evaluating the time-dependent risk of mortality. In order to use longitudinal dialysis condition as a predictive value, laboratory data were not assessed during the second phase. The ESA resistance index (ERI) was calculated by dividing the weekly weight-adjusted ESA dose (IU/kg/ week) by the $\mathrm{Hb}$ concentration $(\mathrm{g} / \mathrm{dL})$, as previously described [12]. Patients were divided into four groups by quartiles (Q) of ERI as follows: Q1 ERI <4.96, Q2 ERI $=4.97-8.30$, Q3 ERI $=8.31-13.1$, and $\mathrm{Q} 4>13.2 \mathrm{IU} / \mathrm{kg} /$ week $/ \mathrm{g} / \mathrm{L}$.

\section{Laboratory and nutrition parameters}

Blood sampling after an overnight fast was performed before the mid-week dialysis session day. Serum urea nitrogen, creatinine, calcium, phosphorous, albumin, total cholesterol, ferritin, transferrin saturation, and Creactive protein (CRP) levels and the $\mathrm{Hb}$ concentration were measured by standard laboratory methods. Total calcium values were corrected by the patient's albumin level. Intact parathyroid hormone (iPTH) was measured by an immunoradiometric assay. Body mass index (BMI) was expressed in kilograms per square meter. Body weight was calculated as dry weight defined as the postdialysis weight of normotensive patients with no signs of overhydration. Urea kinetics were assessed by measuring a blood-based dialysis parameter, Kt/V [13], and the mean value of the three measurements during each of the 3 months before the start of the study was used in the analysis. The normalized protein catabolism rate (nPCR) was used as an indirect indicator of protein intake and was calculated by using the formula previously described [14].

The GNRI was calculated by modifying the nutritional risk index for elderly patients reported by Yamada et al. [5] as follows: GNRI $=[14.89 \times$ albumin $(\mathrm{g} / \mathrm{dl})]+[41.7 \times($ body weight/ideal body weight)]. We used the body weight value at the end of the dialysis session to calculate the BMI. When a body weight exceeded the ideal body weight, body weight/ideal body weight was set equal to 1 [6]. The ideal body weight in the present study was calculated using height and a BMI of 22, which has been reported to be associated with the lowest morbidity rate in the Asian population [15].

\section{Anemia treatment strategy}

Subjects' anemia was treated according to the guidelines published in 2004 by the Japanese Society of Dialysis Therapy (JSDT) [16]. The target $\mathrm{Hb}$ level was $10-11 \mathrm{~g} / \mathrm{dL}$, and epoetin beta was administered to achieve the target $\mathrm{Hb}$ level. Intravenous iron was administered whenever a subject's transferrin saturation was found to be $<20 \%$ or the serum ferritin level was found to be $<100 \mathrm{ng} / \mathrm{mL}$.

\section{Study outcome}

Data for endpoints were obtained from hospital charts and by telephone interviews of patients that were conducted by trained interviewers who were blinded to the study protocol. The primary endpoint of the study was all-cause mortality during the follow-up period from 1 July 2011 to 31 July 2014. The secondary endpoint was cardiovascular death, including death due to heart failure, myocardial infarction, arrhythmia, and stroke. The vital status of the subjects was assessed by searching their electronic dialysis records. Patients were censored if they were alive on 31 July 2014.

Table 2 Predictors of ERI using univariate and multivariate regression analyses

\begin{tabular}{llllll}
\hline & \multicolumn{3}{l}{ Univariate } & & \multicolumn{2}{l}{ Multivariate } \\
\cline { 2 - 3 } \cline { 6 - 7 } & $\beta$ & $p$ value & & $\beta$ & $p$ value \\
\hline Age & 0.140 & 0.0418 & 0.149 & 0.0118 \\
Female vs male & 0.260 & 0.0001 & 0.233 & 0.0003 \\
Dialysis vintage & 0.038 & 0.5373 & 0.032 & 0.5999 \\
Diabetes & -0.013 & 0.8169 & -0.017 & 0.7609 \\
Cardiovascular disease & -0.021 & 0.7035 & -0.013 & 0.8069 \\
KtN & -0.034 & 0.6128 & -0.025 & 0.7009 \\
nPCR & 0.019 & 0.7537 & & \\
Log-CRP & 0.081 & 0.1635 & & \\
Total cholesterol & -0.203 & 0.0010 & -0.194 & 0.0011 \\
Diastolic BP & 0.032 & 0.6049 & & \\
Calcium (corrected) & -0.044 & 0.4237 & & \\
Phosphorus & -0.117 & 0.0391 & -0.105 & 0.0575 \\
Intact-PTH & 0.019 & 0.7430 & & \\
Ferritin & 0.010 & 0.8635 & & 0.0004 \\
TSAT & -0.182 & 0.0023 & -0.193 & \\
GNRI & -0.203 & 0.0007 & -0.205 & 0.0004 \\
\hline
\end{tabular}

Multivariate analysis adjusted for the value for age, gender, dialysis vintage, presence of diabetes and cardiovascular disease, $\mathrm{Kt} / \mathrm{V}$, serum levels of total cholesterol and phosphorus, TSAT, and GNRI

Abbreviations: $n P C R$ normalized protein catabolic rate, BP blood pressure, CRP C-reactive protein, PTH parathyroid hormone, TAST transferrin saturation, GNRI geriatric nutritional risk index 


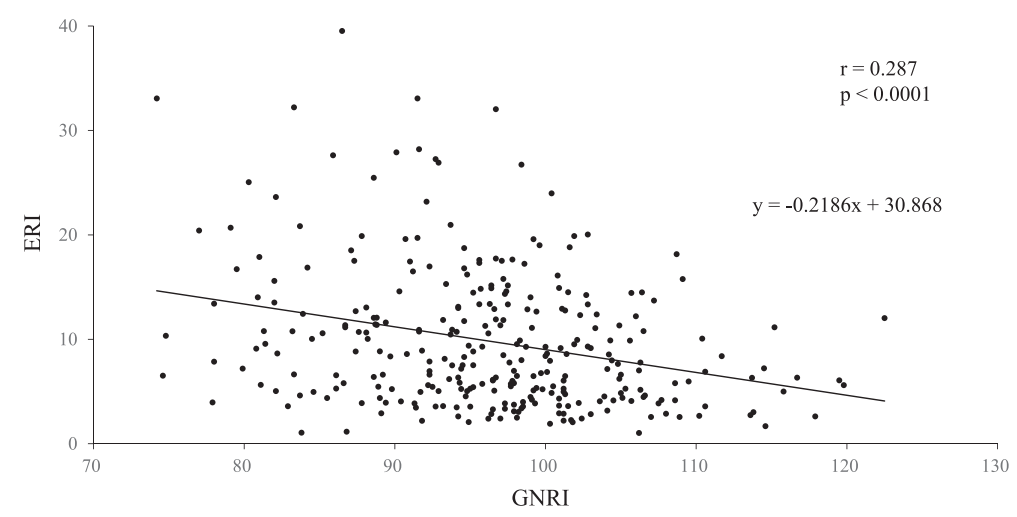

Fig. 1 Scatter diagram showing the association between the GNRI and ERI

\section{Statistical analysis}

Normally distributed, unpaired continuous values were expressed as means \pm SD and compared by performing an analysis of variance. Nonparametric values were expressed as median values and compared by performing the Kruskal-Wallis test. Categorical values were expressed as percentages and compared by performing the Fisher's exact test. Logistic regression analysis was used in a model to determine how independent variables predicted the ERI value. We used Spearman's rank-order correlation coefficients for selected analyses where indicated. Multivariate regression analyses including linear and logistic regression were performed to analyze the data for associations between clinical parameters and ERI. Our fully adjusted model was adjusted for age, gender, dialysis vintage, presence of diabetes, history of cardiovascular disease, Kt/V (single pool), serum total cholesterol and phosphorus levels, transferrin saturation (TSAT), and GNRI.

The survival analysis was based on the Kaplan-Meier curve with subjects censored for death. A log-rank test was used to compare the survival rates of the two groups. Receiver operating characteristic (ROC) analysis was used to select cutoff values of GNRI and ERI for allcause mortality. We considered some variables that possess $P$ value $<0.10$ in univariate logistic regression analysis as independent variables for multivariate analyses. A multivariate logistic regression model with adjustment for multiple factors was used to evaluate mortality risk. Results were expressed as odds ratio (OR) and $95 \%$ confidence interval $(\mathrm{CI})$. A $p$ value of $<0.05$ was considered statistically significant. All statistical analyses were performed by using the SAS version 9.2 software program (SAS Institute Inc., Cary, NC, USA) for Windows personal computers.

\section{Results}

Table 1 shows the baseline characteristics of the 298 HD patients according to their ERI quartiles. The mean age was $66.0 \pm 13.1$ years, $62.8 \%$ were males, $48.3 \%$ were diabetic, and mean dialysis vintage was $5.4 \pm 6.2$ years. Patients with higher ERI were older and had lower GNRI, BMI, Hb, phosphorus, and TSAT. There was a significant relationship between ERI quartiles and both BMI $(p=0.0002)$ and GNRI $(p=0.0003)$.

Table 2 shows the results of the multivariate linear regression analyses between ESA responsiveness and clinical parameters after adjustment for significant confounders by univariate analyses $(p<0.05)$. Both older age $(\beta=0.149)$ and female gender $(\beta=0.233)$ were significant and independent predictors of ERI using multivariate regression analyses. In addition, serum total cholesterol $(\beta=-0.194)$, TSAT $(\beta=-0.193)$, and GNRI $(\beta=-0.205)$ were found to be independent negative predictors of ERI. As shown in Fig. 1, the ERI was inversely correlated with the GNRI $(r=0.287$, $p<0.0001)$.

Table 3 shows the likelihood of ESA at the worst hyporesponsiveness using multivariate logistic regression

Table 3 Likelihood of the worst ESA hyporesponsiveness using multivariate logistic regression analyses to compare the highest versus lowest quartiles of ERI

\begin{tabular}{llll}
\hline & Odds ratio & $95 \% \mathrm{Cl}$ & $p$ value \\
\hline Age & 1.029 & $1.004-1.057$ & 0.0242 \\
Female vs male & 2.385 & $1.193-4.868$ & 0.0138 \\
Dialysis vintage & 1.012 & $0.962-1.064$ & 0.6325 \\
Diabetes & 0.925 & $0.501-1.708$ & 0.8027 \\
Cardiovascular disease & 0.872 & $0.436-1.688$ & 0.6891 \\
Kt $/$ N & 0.631 & $0.148-2.586$ & 0.5227 \\
GNRI (for 1 SD $\uparrow$ ) & 0.681 & $0.494-0.930$ & 0.0155 \\
Total cholesterol (for 1 SD $\uparrow$ ) & 0.643 & $0.456-0.892$ & 0.0077 \\
Phosphorus (for 1 SD $\uparrow)$ & 0.882 & $0.648-1.191$ & 0.4133 \\
TSAT (for 1 SD $\uparrow)$ & 0.582 & $0.415-0.797$ & 0.0006 \\
\hline
\end{tabular}

GNRI geriatric nutritional index, TSAT transferrin saturation 

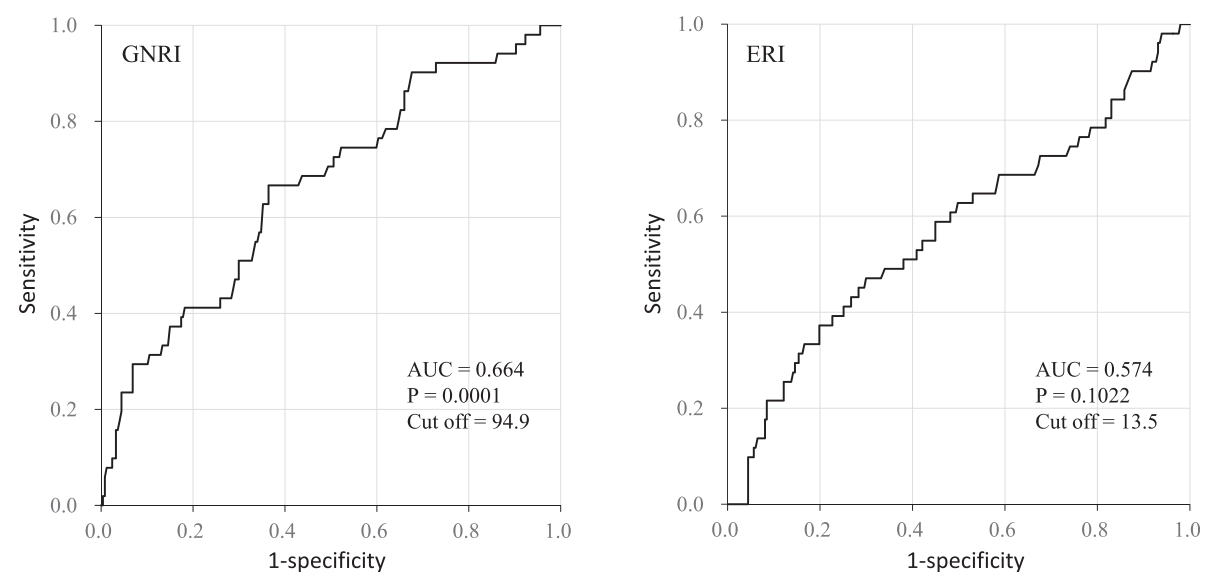

Fig. 2 Receiver operating characteristic analysis showing cutoff values of GNRI and ERI for increased all-cause mortality

analyses to compare the highest (worst) versus three lower quartiles (as reference) of responsiveness to ESA. The likelihood of belonging to the highest ERI quartile in comparison with the three lower quartiles decreased for every $1 \mathrm{SD}$ higher GNRI $(\mathrm{OR}=0.681 ; 95 \% \mathrm{CI}=$ $0.494-0.930)$, every $1 \mathrm{SD}$ higher total cholesterol $(\mathrm{OR}=$ $0.643 ; 95 \% \mathrm{CI}=0.456-0.892)$, and every $1 \mathrm{SD}$ higher TSAT $(\mathrm{OR}=0.582 ; 95 \% \mathrm{CI}=0.415-0.797)$.

The mean follow-up period was $34.6 \pm 6.1$ months. Of the 298 enrolled subjects, 51 died during the follow-up period, and the causes of death were cardiovascular disease in 31 subjects $(60.7 \%)$ and non-cardiovascular diseases including infection and malignancy in 20 subjects (39.3\%).

ROC analyses were performed to select the optimal cutoff values of GNRI and ERI for increased risk of mortality. Figure 2 shows that the cutoff value of GNRI was 94.9 (area under curve $=0.664, p=0.0001$ ) and that of ERI was 13.5 (area under curve $=0.574$, $p=0.1022$ ). When the subjects were stratified according to their ERI and GNRI values into four groups, those who had low GNRI and high ERI were associated with the highest risk of mortality among the four groups (Fig. 3; log-rank $p<0.001$ ).

In an attempt to clarify whether the combination of ERI and poor nutritional status could be a better predictor for all-cause mortality of MHD patients, we examined ORs of the combination factor (four groups stratified by ERI and GNRI) by multivariate analyses and compared the OR of GNRI only. As shown in Table 4, GNRI was an independent predictor of all-cause mortality $(\mathrm{OR}=0.929 ; 95 \% \mathrm{CI}=0.894-0.964 ; p<0.001)$. In addition, low GNRI and high ERI (GLEH) was the worst predictor of all-cause mortality $(\mathrm{OR}=5.325 ; 95 \% \mathrm{CI}=$ 1.888-15.512; $p=0.0016$ ).

Finally, we performed multivariate analyses of allcause mortality using confounders including quartiles of ERI and GNRI. As shown in Table 5, Q1 and Q2

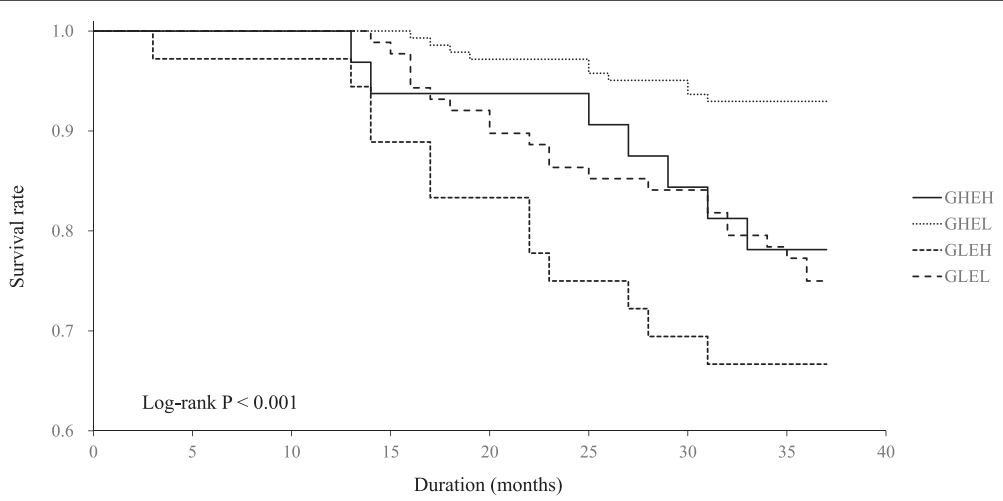

Fig. 3 Kaplan-Meier curve of survival probability according to the GNRI and ERI. High GNRI and ERI high (GHEH) ( $n=32)$; high GNRI and low ERI (GHEL) $(n=142)$; low GNRI and high ERI (GLEH) $(n=36)$; and low GNRI and low ERI (GLEL) $(n=88)$ 
Table 4 Multivariate logistic regression analysis of all-cause mortality according to GNRI and combination factor (four groups stratified by ERI and GVRI)

\begin{tabular}{llll}
\hline Multivariate & Odds ratio & $95 \% \mathrm{Cl}$ & $p$ value \\
\hline Age & 1.052 & $1.016-1.092$ & 0.0039 \\
Male vs female & 2.142 & $1.014-4.771$ & 0.0459 \\
Diabetes & 2.013 & $1.017-4.078$ & 0.0446 \\
Diastolic BP & 0.999 & $0.966-1.034$ & 0.9747 \\
nPCR & 0.944 & $0.140-6.228$ & 0.9521 \\
Ferritin & 1.001 & $0.998-1.004$ & 0.5643 \\
GNRI & 0.929 & $0.894-0.964$ & $<0.0001$ \\
GHEL & 1.000 & & \\
GHEH & 3.234 & $1.036-9.701$ & 0.0436 \\
GLEL & 3.166 & $1.363-7.722$ & 0.0072 \\
GLEH & 5.325 & $1.888-15.512$ & 0.0016
\end{tabular}

GHEH high GNRI and ERI high ( $n=32)$, GHEL high GNRI and low ERI $(n=142)$, GLEH low GNRI and high ERI $(n=36)$, GLEL low GNRI and low ERI $(n=88)$

of GNRI were significant predictors of all-cause mortality, whereas Q3 of ERI only was an independent factor for mortality.

\section{Discussion}

The results of this study showed that ESA responsiveness and nutritional status were associated with increased risk of death after adjustment for potential confounding factors, and, in particular, they showed that patients with high ERI and low GNRI had the highest risk of mortality. These findings suggest that ESA responsiveness and nutritional status should be considered simultaneously when evaluating the relationship between renal anemia and malnutrition and mortality risk in MHD patients.

ESA responsiveness is relative, not absolute, and the two determinants of responsiveness are ESA dose and $\mathrm{Hb}$ level. Previous studies have demonstrated associations between ESA resistance and high all-cause mortality and high cardiovascular mortality in ESRD patients [17-19]. ERI is considered a useful means of ESA resistance, but the follow-up periods in the previous studies were relatively short (i.e., 1 or 2 years). The results of this study showed that a combination of high ERI and low GNRI was associated with increased mortality risk during a mean follow-up period of $34.6 \pm 6.1$ months. Because the ERI and GNRI were calculated from the baseline data, the ERI and GNRI may have changed during the long-term follow-up period.

ESA responsiveness varies widely according to a number of clinical factors. The results of the multivariate logistic regression analysis showed that the likelihood of belonging to the highest ERI quartile in comparison with the three lower quartiles decreased for every 1 SD higher GNRI (OR $=0.681 ; 95 \% \mathrm{CI}=0.494-0.930)$, every $1 \mathrm{SD}$ higher total cholesterol $(\mathrm{OR}=0.643$; $95 \% \mathrm{CI}=0.456-$ $0.892)$, and every $1 \mathrm{SD}$ higher TSAT $(\mathrm{OR}=0.582 ; 95 \%$ $\mathrm{CI}=0.415-0.797)$. These results suggest that nutritional markers are independent predictors of the ESA responsiveness of MHD patients.

Assessment and monitoring of protein and energy nutritional status are essential to prevent, diagnose, and treat uremic malnutrition in dialysis patients [20]. Many nutritional screening tools have been developed for use in the elderly, children, hospitalized patients, patients in the community, and patients with cancer or infections,

Table 5 Multivariate logistic regression analysis of all-cause mortality according to quartiles of ERI or GNRI

\begin{tabular}{|c|c|c|c|c|c|c|}
\hline & \multicolumn{3}{|l|}{ ERI } & \multicolumn{3}{|l|}{ GNRI } \\
\hline & Odds ratio & $95 \% \mathrm{Cl}$ & $p$ value & Odds ratio & $95 \% \mathrm{Cl}$ & $p$ value \\
\hline Age & 1.052 & $1.016-1.092$ & 0.0039 & 1.060 & $1.024-1.100$ & 0.0007 \\
\hline Male vs female & 2.142 & $1.014-4.771$ & 0.0459 & 2.067 & $1.019-4.382$ & 0.0441 \\
\hline Diabetes & 2.013 & $1.017-4.078$ & 0.0446 & 2.032 & $1.034-4.084$ & 0.0395 \\
\hline Diastolic BP & 0.999 & $0.966-1.034$ & 0.9747 & 1.004 & $0.971-1.038$ & 0.8061 \\
\hline $\mathrm{nPCR}$ & 0.944 & $0.140-6.228$ & 0.9521 & 0.850 & $0.125-5.561$ & 0.8659 \\
\hline Ferritin & 1.001 & $0.998-1.004$ & 0.5643 & 1.001 & $0.998-1.005$ & 0.4372 \\
\hline ERI Q1 & 2.165 & $0.809-6.125$ & 0.1248 & & & \\
\hline ERI Q2 & 1.000 & & & & & \\
\hline ERI Q3 & 1.326 & $0.477-3.809$ & 0.5887 & & & \\
\hline ERI Q4 & 2.633 & $1.041-7.162$ & 0.0407 & & & \\
\hline GNRI Q4 & & & & 1.000 & & \\
\hline GNRI Q3 & & & & 2.505 & $0.787-9.636$ & 0.1235 \\
\hline GNRI Q2 & & & & 3.305 & $1.066-12.547$ & 0.0379 \\
\hline GNRI Q1 & & & & 3.492 & $1.132-13.255$ & 0.0287 \\
\hline
\end{tabular}


and some of them can be safely and easily applied to MHD patients as well [21]. The GNRI was one of the parameters that was a predictor of increased overall mortality in the present study. The GNRI is calculated from only a few objective components, including serum albumin and BMI, and is a simple nutritional screening tool [5]. Kobayashi et al. [6] reported finding that HD patients whose GNRI was $<90$ had a poorer nutritional status in terms of lower serum levels of albumin and creatinine than HD patients whose GNRI was $>90$. However, it is very difficult to determine the ideal cutoff value. Park et al. [7] found that GNRI may be a significant predictor of increased mortality in Korean HD patients. More recently, Panichi et al. [22] obtained results closer to those obtained by Yamada et al. and indicated that values below 92 may be a strong indicator of an unfavorable outcome.

Various mechanisms, including derangements in muscle, gastrointestinal, hematopoietic and immune systems, and abnormal activation of the inflammatory process may explain the link between PEW and mortality in renal failure [23]. Low serum albumin and cholesterol levels are indicators of PEW but may not be causally responsible for negative outcome. The reduction in muscle mass, namely sarcopenia, observed in PEW may be due to uremic toxins or procatabolic conditions (metabolic, hormonal, or neuropathic abnormalities). Muscle wasting may in turn lead to reduced skeletal, respiratory, and cardiac muscle function and cause functional insufficiency [24].

The present study had several limitations. First, baseline data were used to define exposure categories in this cohort, and we were unable to examine the effect of changes from the baseline category during the follow-up period. Second, since this was a single center cohort study, the data and results may not be representative of MHD patients in other countries, particularly since the ERI and GNRI are not widely used in Japan. Third, the cohort size was small and the mortality rate was low. However, the follow-up period of our cohort was longer than in previous studies.

\section{Conclusions}

A combination of a high ERI and low GNRI was an independent predictor of increased all-cause mortality in MHD patients. In future studies, greater attention should be focused on background factors that increase responsiveness to ESA in addition to efforts to improve nutritional status.

\section{Competing interest}

The authors have no conflicts of interest to declare.

\section{Authors' contribution}

MO planned the study, searched the literature, assessed studies, extracted data, analyzed data, and prepared article. MK searched the literature, assessed studies, and assisted in article preparation. SS, HK and KT assisted in the data analysis. HK searched the literature and assisted in article preparation. KN planned the study, analyzed the data, and assisted in article preparation. All authors read and approved the final manuscript.

\section{Acknowledgments}

The authors are very grateful to dialysis staff who understood the clinical importance of this study and who provided high-quality data in Jyoban Hospital. This study was in part supported by a grant from Japan Promotion Society for Cardiovascular Disease.

\section{Author details}

${ }^{1}$ Department of Nephrology, Jyoban Hospital, Iwaki City, Fukushima, Japan. ${ }^{2}$ Department of Medicine, Kidney Center, Tokyo Women's Medical University, Shinjuku-ku, Tokyo, Japan.

Received: 3 July 2015 Accepted: 31 August 2015

Published online: 24 November 2015

\section{References}

1. Riella MC. Nutritional evaluation of patients receiving dialysis for the management of protein-energy wasting: what is old and what is new? J Ren Nutr. 2013;23:195-8.

2. de Mutsert R, Grootendorst DC, Axelsson J, Boeschoten EW, Krediet RT, Dekker FW. Excess mortality to interaction between protein-energy wasting, inflammation and cardiovascular disease in chronic dialysis patients. Nephrol Dial Transpalnt. 2008;23:2957-64.

3. Fouque D, Kalantar-Zadeh K, Kopple J, Cano N, Chauveau P, Cuppari L, et al. A proposed nomenclature and diagnostic criteria for protein-energy wasting in acute and chronic kidney disease. Kidney Int. 2008;73:391-8.

4. Lukowsky LR, Kheifets L, Arah OA, Nissenson AR, Kalantar-Zadeh K. Nutritional predictors of early mortality in incident hemodialysis patients. Int Urol Nephrol. 2014;46:129-40.

5. Yamada K, Furuya R, Takita T, Maruyama Y, Yamaguchi Y, Ohkawa S, et al. Simplified nutritional screening tools for patients on maintenance hemodialysis. Am J Clin Nutr. 2008;87:106-13.

6. Kobayashi I, Ishimura E, Kato Y, Okuno S, Yamamoto T, Yamakawa T, et al. Geriatric nutritional risk index, a simplified nutritional screening index, is a significant predictor of mortality in chronic dialysis patients. Nephrol Dial Transplant. 2010;25:3361-5.

7. Takahashi H, Ito Y, IShi H, Aoyama T, Kamoi D, Kasuga H, et al. Geriatric nutritional risk index accurately predicts cardiovascular mortality in incident hemodialysis patients. J Cardiol. 2014;64:32-6.

8. Macdougall IC, Cooper A. The inflammatory response and epoetin sensitivity. Nephrol Dial Transplant. 2002;17 Suppl 1:48-52.

9. Zhang Y, Thamer M, Stefanik K, Kaufman J, Cotter DJ. Epoetin requirements predict mortality in hemodialysis patients. Am J Kidney Dis. 2004;44:866-76.

10. Kilpatrick R, Critchlow C, Fishbane S, Besarab A, Stehman-Breen C, Krishnan $M$, et al. Greater epoetin alfa responsiveness is associated with improved survival in hemodialysis patients. Clin J Am Soc Nephrol. 2008;3:1077-83.

11. Locatelli F, Pisoni R, Combe C, Bommer J, Andreucci VE, Piera L, et al. Anaemia in haemodialysis patients of five European countries: association with morbidity and mortality in the Dialysis Outcomes and Practice Patterns Study (DOPPS). Nephrol Dial Transplant. 2004;19:121-32.

12. Ogawa T, Shimizu H, Kyono A, Sato M, Yamashita T, Otsuka K, et al. Relationship between responsiveness to erythropoiesis-stimulating agent and longterm outcomes in chronic hemodialysis patients: a single-center cohort study. Int Urol Nephrol. 2014;46:151-9.

13. Daugirdas JT. Second generation logarithmic estimates of single-pool variable volume Kt/V: an analysis of error. J Am Soc Nephrol. 1993;4: 1205-13.

14. Shinzato T, Nakai S, Fujita Y, Takai I, Morita H, Nakane K, et al. Determination of Kt/ $\mathrm{V}$ and protein catabolic rate using pre- and postdialysis blood urea nitrogen concentrations. Nephron. 1994;67:280-90.

15. Examination Committee of Criteria for "Obesity Disease"in Japan, Japan Society for the Study of Obesity. New criteria for "obesity disease" in Japan. Circ J. 2002;66:987-92. 
16. Gejyo F, Saito A, Akizawa T, Akiba T, Sakai T, Suzuki M, et al. Japanese Society for Dialysis Therapy. Ther Apher Dial. 2004;8:443-59.

17. Johnson DW, Pollock CA, Macdougall IC. Erythropoiesis-stimulating aganet hyporesponsiveness. Nephrology (Carlton). 2007;12:321-30.

18. Szczech LA, Barnhart HX, Inrig JK, Reddan DN, Sapp S, Califf RM, et al. Secondary analysis of the CHOIR trial epoetin-alpha dose and achieved hemoglobin outcomes. Kidney Int. 2008;74:791-8.

19. Kalantar-Zadeh K, Lee GH, Miller JE, Streja E, Jing J, Robertson JA, et al. Predictors of hyporesponsiveness to erythropoiesis-stimulating agents in hemodialysis patients. Am J Kidney Dis. 2009;53:823-34.

20. Pupin LB, Ikizler TA. Assessment and monitoring of uremic malnutrition. J Ren Nutr. 2004;14:6-19.

21. Kalantar-Zadeh K, Kopple JD, Humphreys MH, Block G. Comparing outcome predictability of markers of malnutrition-inflammation complex syndrome in hemodialysis patients. Nephrol Dial Transpl. 2004;19:1507-19.

22. Park JH, Kim SB, Shin HS, Jung YS, Rim H. Geriatric nutritional risk index may be a significant predictor of mortality in Korean hemodialysis patients: a single center study. Ther Apher Dial. 2012;16:121-6.

23. Panichi V, Cupisti A, Rosati A, Di Giorgio A, Scatena A, Menconi O, et al. Geriatric nutritional risk index is a strong predictor of mortality in hemodialysis patients: data from the Riscavid cohort. J Nephrol. 2014; 2014(27):193-201.

24. Carrero JJ, Stenvinkel P, Cuppari L, Ikizler TA, Kalantar-Zadeh K, Kaysen G, et al. Etiology of the protein-energy wasting syndrome in chronic kidney disease: a consensus statement from the International Society of Renal Nutrition and Metabolism (ISRNM). J Ren Nutr. 2013;23:77-90.

\section{Submit your next manuscript to BioMed Central and take full advantage of:}

- Convenient online submission

- Thorough peer review

- No space constraints or color figure charges

- Immediate publication on acceptance

- Inclusion in PubMed, CAS, Scopus and Google Scholar

- Research which is freely available for redistribution 